\title{
Activation of the Brain During Motor Imagination Task with Auditory Stimulation
}

\section{Long Li}

Xi'an Jiaotong University https://orcid.org/0000-0002-5097-5733

\section{Yanlong Zhang}

Xi'an International Studies University

\section{Liming Fan}

Xian Jiaotong University

Jie Zhao

Xian Jiaotong University

Jing Guo

Xian Jiaotong University

\section{Chenxi Li}

Xian Jiaotong University

Tian Liu

Xi'an Jiaotong University

Jue Wang ( $\nabla$ juewang_xjtu@126.com )

Xi'an Jiaotong University

\section{Research}

Keywords: Auditory stimulation feedback, Mismatch negative (MMN), Inter trial phase locking consistency (ITPC), Electroencephalogram

Posted Date: January 10th, 2022

DOI: https://doi.org/10.21203/rs.3.rs-1136249/v1

License: (c) (1) This work is licensed under a Creative Commons Attribution 4.0 International License.

Read Full License 


\section{Abstract}

Background: Auditory feedback is one of the most important feedback in cognitive process. It plays an important guiding role in cognitive motor process. However, previous studies on auditory stimuli mainly focused on the cognitive effects of auditory stimuli on cortex, while the role of auditory feedback stimuli in motor imagery tasks is still unclear.

Methods: 18 healthy subjects were recruited to complete the motor imagination task stimulated by meaningful words and meaningless words. In order to explore the role of auditory stimuli in motor imagination tasks, we studied EEG power spectrum, frontal parietal mismatch negativity (MMN) and inter test phase-locked consistency (ITPC). one-way Analysis of Variance (ANOVA) and Least Significant Difference (LSD) correction were used to test the differences between the two experimental groups and the differences of different bands in each experimental group.

Results: EEG power spectrum analysis showed that the activity of contralateral motor cortex was significantly increased under the stimulation of meaningful words, and the amplitude of mismatch negative wave was also significantly increased. ITPC is mainly concentrated in $\mu$, $a$ and $\gamma$ bands in the process of motor imagery task guided by the auditory stimulus of meaningful words, while it is mainly concentrated in the $\beta$ band under the meaningless words stimulation.

Conclusions: This results may be due to the influence of auditory cognitive process on motor imagery. We speculate that there may be a more complex mechanism for the effect of auditory stimulation on the inter test phase lock consistency. When the stimulus sound has the corresponding meaning to the motor action, the parietal motor cortex may be more affected by the prefrontal cognitive cortex, thus changing its normal response mode. This mode change is caused by the joint action of motor imagination, cognitive and auditory stimuli. This study provides a new insight into the neural mechanism of motor imagery task guided by auditory stimuli, and provides more information on the activity characteristics of the brain network in motor imagery task by cognitive auditory feedback.

\section{Background}

Sensory processing is one of the important functions of the human body. Our senses can provide us with a continuous flow of information, which enables us to obtain the constantly changing environmental information around us. The input of environmental information plays an important role when a human completes some sports tasks. For example, when people hold an egg, the input of tactile information can enable us to obtain pressure information. Based on the pressure information, we can control our hands to hold the egg with appropriate strength so that it neither slips because of too little strength nor breaks because of too much strength ${ }^{[1]}$. For daily motion tasks, vision, hearing and touch are the most important input of information flow to complete motion control. Amongst them, there are obvious differences between auditory information input and the other two kinds of information input. Vision and touch belong to the intuitive information flow, they can make us intuitively understand the position and strength of 
objects and other information, while auditory input is often accompanied by prior knowledge learning. After receiving some meaningful or preset auditory information input, the brain will process it at the cognitive level. For example, the rhythmic sound stimulation ${ }^{[2,3]}$, which is widely used in the rehabilitation of the lower limbs, will let the patients move the lower limbs rhythmically according to the specific prompt sounds in the treatment process. In fact, these prompt sounds are given special significance. After the patients hear the syllable prompts, the brain will carry out cognitive processing analysis on them, which is also the unique feature of auditory stimulation ${ }^{[4]}$. Auditory stimuli can be divided into the following types according to the content of the information package: (1) pure sound without meaning, such as noise, background sound, etc.; (2) environmental sound, such as finger knocking, footsteps, etc.; (3) semantic sound with meaning.

At present, the research on auditory stimulation is mainly based on ERP. In the study of auditory ERP, mismatch negativity (MMN) can be used to detect some discernable changes caused by auditory stimuli, which is a reliable test method ${ }^{[5,6]}$. Previous studies have extended the concept of MMN to higher-level cognitive processes, such as those involving semantics, which may have different response patterns in sound stimulation ${ }^{[7]}$. Therefore, in order to explore the effect of sound stimulation on motor intention, we need to know the difference of response mode between pure tone stimulation and meaningful words stimulation in human brain. The researchers found that there are different response patterns for pure tone stimulus and meaningful words. At present, the common conclusion is that pure tone is mainly recognized in the right hemisphere, while meaningful tone is recognized in the left hemisphere. Satoko Asano studied whether segmented speech is regarded as a time unit like pure tone, and whether there is difference between left and right ear stimulation. 25 right-handed men were used to detect the time integral of segmented speech by using a series of mismatched negative abnormal sounds in standard speech without paying attention to it, and unilateral and bilateral speech stimulation were performed respectively ${ }^{[8]}$. They found that all bilateral stimuli induced clear MMN with similar peak latency. At the same time, the amplitude of MMN was significantly different between pure tone stimulus and speech stimulus. Michael Weigl found that the left frontal anode tDCS could reduce the duration and intensity deviation of MMN by stimulating the subjects' left prefrontal dorsolateral cortex with tDCS, which further highlighted the contribution of frontal lobe brain area to MMN production ${ }^{[9]}$. In addition, MMN data show that $\mathrm{MMN}$ can show people's activation and attention conversion after receiving auditory stimulus stream, and can establish brain response process. Previous studies have also explored the effect of sound stimulation on motor function. 0 . Huak explored the activation dynamics of human motion recognition system. They studied the electrophysiological differences between the brain response to the sound produced by human finger movement ${ }^{[10]}$. They found that finger induced clicks produced more MMN than control stimuli. This shows that the sound stimulation related to movement can activate the memory track of familiar sound related to movement. In addition, 0 . hauk found that the MMN map of this incubation period showed a difference in brain response to natural finger and natural tongue sounds. Sound source estimation revealed that the click caused by fingers originated from the left hemisphere. The results show that: (1) the production of $M M N$ is related to the process of bilateral temporal response and frontal parietal response; (2) external intervention can change the response mode of MMN; (3) 
hearing the environmental sound related to movement will arouse the activity of the movement area related to the dominant hand. Generally speaking, most of the previous studies focused on the activation of MMN effect by sound stimulation, while few people studied the effect of sound stimulation on human motor function.

Previous ERP studies on auditory stimulation suggested that some special frequency band concussions were caused by the noise of EEG acquisition system or uncontrollable interference source. However, recent studies have found that in some special frequency bands, such as $a, \beta$, and $y$ bands, the ongoing EEG oscillations are also related to stimulation related brain activities, which cannot be simply considered as "noise" [11,12]. Therefore, the use of ERPs alone in cognitive research may ignore some valuable information, such as the phase of oscillation in EEG. In order to solve this problem, researchers have developed several tools ${ }^{[13,14]}$ to analyze the ongoing EEG dynamics, one of which is the inter-trial phaselocking consistency (ITPC), also known as "phase locking factor". ITPC is a synchronous time-frequency measurement method related to specific experimental stimuli. It can be used to explore the relationship between EEG evoked potential and spontaneous EEG oscillation phase ${ }^{[15]}$, and predict some cognitive processes. Goran Papenberg et al ${ }^{[16]}$. Found that the consistency of ITPC increased gradually from childhood to early adulthood, and then decreased from early adulthood to old age by comparing the ITPC between young and old people at low theta frequency band. Luodi Yu et al ${ }^{[17]}$. Found that the components of auditory evoked potential driven by ITPC may coexist according to the different stages of information processing, which shows that ITPC plays a very important role in typical auditory evoked potential experiments, and it plays the role of amplitude prediction factor and difference discrimination factor.

Although there have been many studies on the effect of auditory stimulation on human cognitive function, the mechanism of auditory stimulation as feedback of motor task is not clear ${ }^{[18]}$. At the same time, the previous research methods are limited to single time domain or frequency domain phase research, and the correlation between time domain information and frequency domain phase information still lacks of exploration [19]. Therefore, we hope to further study how sound stimulation interferes with the neural mechanism of human motor function response. Based on the behavioral experiments of auditory stimulation and the above MMN and ITPC studies, we hypothesized that the auditory stimulation including meaningful (movement command) can affect the feedback control loop of brain in the motion imaging task, and the input of sound motion command stimulation can change the activation mode of the senior cortex in the motion imaging task. In order to test this hypothesis, we try to analyze the relationship between temporal primary auditory cortex and motor sensory cortex in the process of auditory input and determine whether there are dominant factors. First of all, we use the traditional power spectrum topographic map method to obtain the brain's regions activated by auditory stimulation under the motion imagination task. After determining the activation area, we used MMN method to study the effects of meaningful and meaningless auditory stimuli on the response patterns of primary auditory cortex and advanced motor cortex of temporal lobe in the motor imagination task. Then, in order to study the difference mechanism, we use ITPC method to obtain the frequency domain phase information in the 
response process, and analyze the possible sources of the difference, in order to verify the neural mechanism explanation of the effect of meaningful words stimulation on brain activation mode. Based on our findings, we infer that appropriate auditory training instruction in rehabilitation training may change the activation mode of brain area. Therefore, this study can provide a new treatment for the rehabilitation of the patients with motor disorders after stroke and other brain injury diseases.

\section{Materials And Method}

\subsection{Participants}

Eighteen healthy right-handed volunteers (mean age,22; range,19-24; 6 males and 12 females), with normal auditory function participated. All subjects' semantic understanding is normal. All the subjects had signed informed consent and approval of the local ethics committee.

\subsection{Experimental paradigm}

Oddball paradigm is one of the classical paradigm in auditory stimulation, which can induce P300, MMN and other ERP components related to stimulation probability. In this experiment, the subjects in the nonattention state were continuously presented with standard stimulus and deviation stimulus, the probability of which was significantly different. Amongst them, the standard stimulus was pure tone stimulus, the tone length of which was the same as that of deviation stimulus, accounting for $90 \%$ of the total stimulus; the deviation stimulus was the meaningful "grasp" or meaningless word, accounting for $10 \%$ of the total stimulus, which was pseudo-random. The first five stimuli did not include biased stimuli, and at least five standard stimuli were designed between each two biased stimuli. At the beginning of the experiment, the subjects were asked to sit at the table in a comfortable way with their arms flat in front of the table. During the experiment, a cup will be placed in front of the right hand of the subject, and the subject is required to complete the movement imagination task of the right hand grasping when hearing the deviation stimulus "grasping." During the whole experiment, the subjects are required to look at the screen and see the cup in the residual light. In this way, in order to eliminate the influence of attention factors on the experimental results, the subjects can complete the grasping motion imagination task. The experiment consisted of two tasks: 'M-ST' and 'N-ST'. Amongst them, 'M-ST' meant the deviation stimulus is the word with meaning of "grasp," and 'N-ST' meant the deviation stimulus is the word without meaning.

After the start of the experiment, each group contained 400 stimuli, which were played automatically according to the oddball paradigm. The interval between the stimuli was $800 \mathrm{~ms}$, and each group was rested for 5 minutes after the end of the experiment. During the broadcast, the subjects wore monitoring headphones (iron triangle ath-m50x) and watched a silent movie (Rescued Ginger, directed by Quentin Tarantino, 2012; or Encoffiner, directed by Yoshiro OTA, 2008). Both are played randomly). The selected clip avoids the more intense scenes such as plot and lens. The subjects were required to maintain emotional stability, focus on the film, ignore the sound stimulation, avoid blinking and body movement as much as possible, allow short rest and adjust the earphone and other small movements between groups, 
and ask the subjects about the summary of film clips after the experiment to confirm their concentration. The overall program is shown in Figure 1 - (a) and the actual scene is shown in Figure 1 - (b). The design and implementation are implemented by MATLAB software.

\subsection{Recordings}

The experiment was performed in an electromagnetic shielding room. EEG data was recorded with a SynAmps ${ }^{2}$ system (Neuroscan, El Paso, TX, USA). An elastic cap with 32 mounted Ag/ $\mathrm{AgCl}$ electrodes was positioned according to the extended 10-20 system which was used to detect EEG activities with the reference at the vertex. The EEG electrode impedances were kept below $5 \mathrm{~K} \Omega$ during the whole experiment. All data were referenced against an electrode centered on the midline between Fz and Cz. EEG data were band-pass filtered $(0.5-100 \mathrm{~Hz})$. All signals were sampled to disk at $1000 \mathrm{~Hz}$ together with event markers and saved on a hard disk for off-line analysis.

\subsection{Data analysis}

During the experiment, playing audio through MATLAB completes the alignment with EEG signal at the same time. Firstly, EEG is preprocessed by using the EEG toolbox, which mainly completes the $0.5-50 \mathrm{hz}$ band-pass filtering, which contains all the band information needed to be observed in this experiment. Then independent component analysis (ICA) is performed to remove the components with dominant artifacts (such as blink, eye movement, muscle artifacts, heartbeat). After pre-processing by the EEG toolbox, the EEG data is transformed into the data structure applicable to the field trip toolbox through the built-in interface function between the EEG toolbox and the field trip for subsequent processing and calculation.

To capture the difference in motor sensory cortex during the action execution period and moment stabilization stage in different brain rhythms, power, MMN, and ITPC were estimated pair-wise between all EEG channels. Our study mainly used an open-source toolbox, Field-Trip ${ }^{[20]}$, for neurophysiological data analyzing.

\section{5 Power estimated of EEG}

The power spectrum of the electroencephalogram (EEG) signal of the interested electrode was calculated by using the multi-taper method, and the power of EEG was normalized to the sum of the total power of all frequencies (from $0 \mathrm{~Hz}$ to $500 \mathrm{~Hz}$ ). This is an estimate of the power ratio for a given frequency band. In the $1200 \mathrm{~ms}$ test data, the EEG data after sound stimulation (200ms after the start of the experiment) were used for further analysis. After grabbing the concerned data segments, the obtained data are divided into $\alpha / \mu$ rhythms (5-14hz), $\beta$ rhythms (14-35hz) and low $\gamma$ rhythms (35-45hz) according to brain rhythms. When EMG signal or other artificial noise pollutes EEG signal, all EEG data segments are excluded artificially.

\section{6 Estimation of MMN}


The calculation of MMN is actually the difference wave obtained by subtracting the ERP signal generated by standard stimulus from the ERP signal generated by deviating stimulus. In general, the peak value of MMN occurs between $150-200 \mathrm{~ms}$. In this paper, the starting time of acoustic stimulation is taken as the data starting point, and the data $200 \mathrm{~ms}$ after the start of acoustic stimulation is taken as the analysis time process. After data collection, the biased stimulus sequence and the standard stimulus sequence were overlapped and averaged respectively. Finally, the MMN value is obtained by calculating the difference of the mean value.

\section{7 Estimation of ITPC}

Trial-by-trial time-frequency analysis was carried out in EEGLAB (Delorme et al., 2004). Inter-trial phase coherence (ITPC) was computed using the "newtimef" function:

$$
\operatorname{ITPC}(\mathrm{f}, \mathrm{t})=\frac{1}{n} \sum_{k=1}^{n} \frac{F_{k}(f, t)}{\left|F_{k}(f, t)\right|}
$$

\section{In this function, $F_{k}(f, t)$ is the spectral estimate of trial $\mathrm{k}$ at frequency $\mathrm{f}$ and time}

\section{t obtained using short-time Fourier transformation (STFT), and $\|$ represents the}

complex norm of trial k. The modified STFT (with Hanning tapers) in EEGLAB uses overlapping sliding windows that are adaptive to the target frequency bins (i.e., the time window decreases linearly as frequency increases), which is recommended to overcome limitations of conventional fixed window in estimating low frequency activities. The frequency range analyzed was $0.5-50 \mathrm{~Hz}$. Zero-padding was applied to windows without sufficient number of sample points with a pad-ratio of 16 with a frequency spacing of $0.5 \mathrm{~Hz}$. ITPC value of a given frequency at a given time point can range from 0 to 1 . Larger ITPC values indicate higher phase consistency across trials, and smaller values indicate lower consistency or larger neural "jittering". For the calculation of theta ITPC, the ITPC data were first averaged across the frequencies for further processing [21].

For this study, we not only pay attention to the influence of syllable stimulation in time domain, but also to the characteristics of induced phase locking, which represents the time locking and induced components in the meaningful words stimulus. Therefore, we calculated inter-trial phase coherence (ITPC) and converted it into Rayleigh Z score to explain the difference between the M-ST group and the NST group.

\section{8 Statistical analysis}

In order to evaluate our hypothesis, the statistical analysis process is divided into three steps. First of all, the differences of EEG power spectrum between the stimulus task of M-ST group and that of N-ST group 
were compared by one-way ANOVA. In order to compare the MMN changes of M-ST group and N-ST group, we used one-way AVOVA and LSD correction to test the MMN differences of the two experimental groups and the differences of different wave bands in each experimental group. The third step of statistical analysis is to use one-way ANOVA in different frequency bands to verify the difference between the M-ST and N-ST groups.

\section{Results}

\subsection{Topographic map of EEG power spectrum}

By comparing the distribution characteristics of EEG power and power spectrum map under the stimulation of meaningless words and meaningful words, we can determine whether the meaningful words stimulation has a differential effect on the brain activity under the exercise imagination task. We divided the data into $a$ band, $\beta$ band and $y$ band to analyze the activity characteristics of primary auditory cortex and advanced motor cortex in different frequency bands. As shown in the figure [Fig. 2, Fig. 7], we found that the main areas of brain activation in the motor imagination task under auditory stimulation are left temporal lobe and frontal parietal lobe. Under the stimulation of real meaning the activation range of the left frontal parietal lobe was significantly stronger than that of the meaningless stimulus, within the band of beta and gamma $(F=4.512 p=0.041, F=5.508 p=0.031)$ [Table.1]. At the same time, the activation level of cognitive cortex in the real meaning sound stimulation group was higher than that in the meaningless sound stimulation group $(F=4.561 \mathrm{p}=0.040, \mathrm{~F}=5.002 \mathrm{p}=0.032)$ [Table.1].

\subsection{Mismatch negativity (MMN)}

We use the traditional power spectrum mapping method to determine that the main brain areas activated by auditory stimulation in motor imagery task are frontal parietal lobe, bilateral temporal lobe and prefrontal lobe. MMN data induced by biased stimulus were recorded and calculated on FCZ of frontal midline electrode in the M-ST and N-ST groups. On this basis, the following two factor variances are carried out for amplitude and latency respectively. As shown in the figure [Fig. 3], the time-domain distribution of the mean $M M N$ of eighteen subjects can be seen from the figure that two MMN peaks are generated within $100-300 \mathrm{~ms}$, which are located near $150 \mathrm{~ms}$ and $250 \mathrm{~ms}$ respectively. At the same time, it can also be found that the peak value of MMN produced by meaningful words stimulation is earlier than that of meaningless words in time axis. Because of the difference in the time of peak occurrence, we use the maximum value of MMN produced by meaningful words stimulation or meaningless words stimulation in $100 \mathrm{~ms}-300 \mathrm{~ms}$ as the center, and take the average value of the sum of the values within $10 \mathrm{~ms}$ from the center as the statistical calculation value.

In this paper, one-way ANOVA was used to evaluate the effect of M-ST group and N-ST group on MMN amplitude. As shown in the table [Table.2] and the figure [Fig. 8], the MMN amplitude caused by "real meaning sound stimulus" is significantly higher than that caused by "meaningless sound stimulus" $(F=4.495 \mathrm{p}=0.04)$. 


\subsection{Inter-trial phase coherence (ITPC)}

As a time-frequency measurement method, ITPC is mainly used to explore the relationship between EEG evoked potential and the phase of spontaneous EEG oscillation, and to show the auditory related cognitive process. As shown in the figure [Fig. 4, Fig. 9], in the beta and low gamma frequency bands, the distribution of ITPC is basically the same under the standard stimulation of M-ST and N-ST group, while the distribution of ITPC under the deviation stimulation is significantly different [Table.3]. According to the distribution of EEG power spectrum, we mainly focus on the characteristics of ITPC in prefrontal and parietal motor cortex. The experimental results showed that the intra group phase coupling of prefrontal cortex showed a high level under the stimulation of meaningful words, while the intra group phase coupling of parietal motor cortex and contralateral motor cortex showed a significant frequency band differential distribution [Fig. 4, Fig. 5]. It is characterized by the fact that ITPC is mainly distributed in the low gamma band and $\mu$ band under the stimulation of meaningful words, while ITPC is mainly distributed in the beta band under the stimulation of meaningless words. At the same time, the ITPC value of the left temporal lobe is higher than that of the right temporal lobe [ Fig. 6].

\section{Discussion}

Based on the study of EEG power spectrum in the process of motor imagery task, we found that the right hand motor imagery task induced by auditory stimulation can cause a greater power response in the left parietal lobe (C3). This phenomenon is in line with the expectation of the experiment, and also consistent with the previous research results on the motion imagination task ${ }^{[22,23]}$. At the same time, we compared the power intensity of the left and right motor cortex, the contralateral auditory temporal cortex and the prefrontal cognitive cortex under the stimulation of meaningless words and meaningful words, and found that the activation intensity of the left motor cortex under the stimulation of meaningful words was significantly higher than that of meaningless words. Similar findings have been found in other similar studies focusing on cortical activation. For example, BOE. S et al. Pointed out in a study of visual guided motor imagery tasks in 18 healthy subjects that repeated motor imagery and neural feedback can produce lateralized effects on brain activity, and can improve the motor imagery task hand under the guidance of visual cues activation degree of contralateral motor cortex ${ }^{[24]}$. Glover et al. studied visual stimulus guidance, context stimulus (visual context) guidance, and digital stimulus guided motor imagery tasks. They found that after removing visual guidance, context variables play a greater role in motor imagery tasks and behavior performance. This shows that context cognition plays an important role in sports imagination ${ }^{[25]}$. Based on the previous research results, we conclude that the motor imagination task under the stimulation of the meaningful words can enhance the activation intensity of the contralateral motor brain area similar to the visual stimulation. Compared with the research of Glover et al., the contextual stimuli used in our study come from auditory stimuli. At the same time, in our experiment, we also found that the prefrontal cognitive cortex is always in a high level of activated water products. Therefore, we speculated that visual and auditory contexts can guide the subjects to complete the task of motor imagination by affecting the cognitive cortex. 
Previous studies have extended the concept of MMN to a higher-level cognitive process, such as the cognitive process involving semantics. Semantics may have different response patterns in sound stimulation. Therefore, in our experiment, we also studied the MMN amplitude intensity to explore the effect of sound stimulation on motor intention. The experimental results show that the MMN responses of both the M-ST and the N-ST groups are significant around 250ms, and the MMN amplitude of the meaningful words stimuli is significantly higher than that of the meaningless words stimuli. Hauk et al. studied MMN induced by environmental sound stimulation related to finger movement and found that hearing the sound related to finger movement can arouse the activity of the motion area related to dominating fingers ${ }^{[10]}$. According to the research data of Hauk et al., the motor regions in the human brain are part of the early automatic recognition of action related sounds. Lepage had come to a similar conclusion. They believe that there is a rapid, multimodal resonance mechanism to regulate the activity of motor cortex after receiving sound stimulation. MMN is caused by any discernible change in a repetitive aspect of sustained auditory stimulation ${ }^{[26]}$. Even without attention, MMN can also cause the change of attention transfer, so it shows a significant response to the body. It is important that MMN reflects the function of NMDA receptor ${ }^{[27]}$. The improvement of MMN can provide an effective index for the treatment of brain injury ${ }^{[28]}$. The experimental results show that the MMN peak value produced by the meaningful stimulus is more advanced in the time axis than that produced by the meaningless stimulus, and the average amplitude is also significantly higher than that produced by the meaningless stimulus. Therefore, we believe that under the repeated meaningful words stimulus, it is more likely to cause the shift of attention of the subjects, making the subjects focus more on the movement imagination task, at the same time, the meaningful words stimulation has the similar function with the environment sound stimulation used by Huak et al. It can also arouse the brain activity related to the hand movement.

Stroke and other neurological diseases have adverse effects on cognitive and motor functions. These damages are related to the changes of EEG power spectrum and event-related potential measurements. However, the effect of auditory stimulation on phase congruence (ITPC), a method to measure phase congruence during the experiment, is still unknown. ITPC is considered to reflect the ability of nerve response and related events to synchronize in time, so as to optimize information processing. In this study, we found that the phase consistency of auditory guided motor imagery evoked potential in different frequency bands of different brain regions showed a certain regularity in the motor imagery task under the stimulation of meaningful words compared with that under the stimulation of meaningless words. Our results showed that the ITPC in theta, a and low gamma rhythms increased significantly when the subjects were asked to perform motor imagery task under the effect of real meaning sound stimulation. The ITPC frequency band differences in motion regions indicate that the task of motion imagination guided by sound stimulation is a complex "stimulus cognitive motion" feedback process, in which semantic recognition plays an important role. The increase of ITPC in the parietal motor cortex may be caused by attention or sensory stimulation, which was also found in previous studies on the effect of tactile stimulation on motor tasks. At the same time, the prefrontal cortex showed higher activation of ITPC under the stimulation of meaningful words, which indicated that the cognitive cortex could participate more in the activities of motor imagination tasks under the stimulation of meaningful 
words, and this stimulation improved the synchronization ability of prefrontal and sound stimulation events, which was completely consistent with the results of MMN. Papenberg studied the difference of ITPC in theta / alpha frequency band in different age groups and found that ITPC in theta / alpha frequency band can represent the change of response time variability to stimulation, that is to say, the faster the response time, the higher the ITPC ${ }^{[29]}$. Yu $L$ also had similar research. They found that the ITPC amplitude of theta / alpha band was related to the decrease of auditory evoked response after the subjects were given verbal and non-verbal stimulation respectively. In other words, verbal stimulation can improve the auditory evoked response ${ }^{[30]}$. Combined with previous studies, we believe that the meaningful words stimulus can improve the response efficiency related to the exercise task in the exercise imagination task, that is to say, the meaningful words stimulus can reduce the response time of the subjects and improve the efficiency of the exercise imagination task. In the experiment, we found another interesting phenomenon: ITPC increased significantly in beta rhythm under the stimulation of no meaningful words, while ITPC showed a lower level in beta rhythm under the stimulation of meaningful words. This phenomenon has not been mentioned in similar studies. We speculate that this may be due to the lack of synchronization of cognitive process activated by nonsense stimulation, which results in the brain activity mainly concentrated in the parietal motor cortex during the task, and the main active rhythm in the traditional motor imagination task is beta rhythm.

The results showed that the ability of the brain to synchronize with the rhythmic stimulus was improved by the stimulation of the meaningful words. Therefore, the rise of ITPC may be an objective and mechanical measurement method of motor imagination function, which enables future work to study the relationship between brain world synchronization and specific brain function activation related to motor tasks.

\section{Conclusion}

This study has some limitations. First of all, we hope to understand the effect of auditory stimulation on motor function. Only from the results of motor imagery task, the real meaning sound stimulation can effectively improve the brain response mode, but whether the real meaning sound stimulation has similar results for the motor task remains to be verified. Therefore, in the future, we plan to carry out further research to confirm whether this improvement exists in motor tasks. Secondly, sample size is also a key limitation of our study. Small sample size may limit the statistical results and provide little classification information.

\section{Declarations}

Ethics approval and consent to participate: The author promised that all subjects had signed the informed consent form and obtained the approval of the local ethics committee.

Consent for publication: All authors agree to publish the article in the Journal of NeuroEngineering and Rehabilitation. 
Availability of data and materials: The datasets used or analysed during the current study are available from the corresponding author on reasonable request.

Competing interests: The authors declare that they have no competing interests.

Funding: This work was supported by a a National Natural Science Foundation of China (Grant No. U1913216) a National Natural Science Foundation of China (Grant No. 61431012), a National Key Research and Development Program of China (No.2017YFB1300303, 2018YFC2002601).

Author details: 1.The Key Laboratory of Biomedical Information Engineering of Ministry of Education, The Key Laboratory of Neuro-informatics \& Rehabilitation Engineering of Ministry of Civil Affairs, and Institute of Health and Rehabilitation Science, School of Life Science and Technology, Xi'an Jiaotong University, Xi'an 710049, P. R. China. 2.National Engineering Research Center of Health Care and Medical Devices. 3.Xi'an International Studies University, Xi'an 710128, P. R. China.

Authors' contributions: LL participated to study design, data collection and analysis, and manuscript writing; YLZ participated to study design, data collection and analysis, and manuscript definition; LMF participated to data analysis; JZ participated in the data collection; JG participated to study design; CXL participated to data collection and neurophysiological interpretation; TL participated to study design; JW participated to study design; All authors read and approved the final manuscript.

Acknowledgements: The author thanks Dr. Yanlong Zhang for his help in subject recruitment and syllable stimulation, as well as Professor Tian Liu, Professor Jue Wang and Dr. Liming fan for their help in the revision of the article. At the same time, I would like to thank National Natural Science Foundation of China (Grant No. U1913216) for its financial assistance.

\section{References}

1. Li L, Guo J, Zhang Y, Wu H, Li L, Liu T, et al. Pattern Reorganization of Corticomuscular Connection with the Tactile Stimulation. Annals of Biomedical Engineering 2020; 48(2):834-847.

2. J-H, Song, P-Y, Zhou, Z-H, Cao, et al. Rhythmic auditory stimulation with visual stimuli on motor and balance function of patients with Parkinson's disease. European Review for Medical \& Pharmacological Sciences 2015.

3. Massimiliano P, Federica C, Roberta P, Carlo C, Fabrizio S, Tiziano A, et al. Effects of Physical Rehabilitation Integrated with Rhythmic Auditory Stimulation on Spatio-Temporal and Kinematic Parameters of Gait in Parkinson's Disease. Frontiers in Neurology 2016; 7(3).

4. Thaut MH, Leins AK, Rice RR, Argstatter H, Kenyon GP, Mcintosh GC, et al. Rhythmic Auditor y Stimulation Improves Gait More Than NDT/Bobath Training in Near-Ambulatory Patients Early Poststroke: A Single-Blind, Randomized Trial. Neurorehabil Neural Repair 2007; 21(5):455-459. 
5. Näätänen R, Jacobsen T, Winkler IN. Memory-based or afferent processes in mismatch negativity (MMN): A review of the evidence. Psychophysiology 2010; 42(1):25-32.

6. Näätänen $R$, Paavilainen $P$, Rinne $T$, Alho $K$. The mismatch negativity (MMN) in basic research of central auditory processing: A review. Clinical Neurophysiology 2007; 118(12):2544-2590.

7. Tsolaki AC, Kosmidou V, Kompatsiaris I, Papadaniil C, Hadjileontiadis L, Adam A, et al. Brain source localization of MMN and P300 ERPs in mild cognitive impairment and Alzheimerl"s disease: a highdensity EEG approach. Neurobiology of Aging 2017:S019745801730101X.

8. Asano S, Shiga T, Itagaki S, Yabe H. Temporal integration of segmented-speech sounds probed with mismatch negativity. Neuroreport 2015; 26(17):1061-1064.

9. Weigl M, Mecklinger A, Rosburg T. Transcranial direct current stimulation over the left dorsolateral prefrontal cortex modulates auditory mismatch negativity. Clinical Neurophysiology 2016; 127(5):22632272.

10. Hauk O, Shtyrov Y, Pulvermüller F. The sound of actions as reflected by mismatch negativity: rapid activation of cortical sensory-motor networks by sounds associated with finger and tongue movements. European Journal of Neuroscience 2006; 23(3).

11. Engel AK, Fries P, Singer W. Dynamic predictions: Oscillations and synchrony in top-down processing. Nature Reviews Neuroscience 2001; 2(10):704-716.

12. Jansen $B H$, Agarwal G, Hegde A, Boutros NN. Phase synchronization of the ongoing EEG and auditory EP generation. Development \& Change 2003; 114(1):79-85.

13. Blankertz B, Tomioka R, Lemm S, Kawanabe M, Muller KR. Optimizing Spatial filters for Robust EEG Single-Trial Analysis. 2008.

14. Debener S, Ullsperger M, Siegel M, Engel AK. Single-trial EEG-fMRI reveals the dynamics of cognitive function. 2006.

15. Busch NA, Dubois J, Vanrullen R. The phase of ongoing EEG oscillations predicts visual perception. 2009.

16. Papenberg G, Ferencz B, Mangialasche F, Mecocci P, Cecchetti R, Kalpouzos G, et al. Physical activity and inflammation: effects on gray-matter volume and cognitive decline in aging. Human Brain Mapping 2016.

17. Huang $D, Y u L$, Wang $X$, Fan $Y$, Zhang $Y$. Distinct patterns of discrimination and orienting for temporal processing of speech and nonspeech in Chinese children with autism: An event-related potential study. European Journal of Neuroscience 2018; 47(662):668. 
18. Dijk EO, Nijholt A, Erp JBFV, Wolferen GV, Kuyper E. Audio-tactile stimulation: a tool to improve health and well-being? International Journal of Autonomous \& Adaptive Communications Systems 2013; 6(4):305-323.

19. Paavilainen P, Simola J, Jaramillo M, R N, Winkler I. Preattentive extraction of abstract feature conjunctions from auditory stimulation as reflected by the mismatch negativity (MMN).

Psychophysiology 2010; 38(2):359-365.

20. Oostenveld R, Fries P, Maris E, Schoffelen JM. FieldTrip: Open Source Software for Advanced Analysis of MEG, EEG, and Invasive Electrophysiological Data. Computational Intelligence \& Neuroscience 2011; 2011(3):156869.

21. Luodi, Wang, Suiping, Huang, Dan, Xueyuan, et al. Role of inter-trial phase coherence in atypical auditory evoked potentials to speech and nonspeech stimuli in children with autism. Clinical neurophysiology 2018.

22. Burianova H, Sowman PF, Marstaller L, Rich AN, Williams MA, Savage G, et al. Adaptive Motor Imagery: A Multimodal Study of Immobilization-Induced Brain Plasticity. Cerebral Cortex 2014:bhu287.

23. Tabrizi YM, Mazhari S, Nazari MA, Zangiabadi N, Sheibani V, Azarang S. Compromised motor imagery ability in individuals with multiple sclerosis and mild physical disability: An ERP study. Clin Neurol Neurosurg 2013; 115(9):1738-1744.

24. Boe S, Gionfriddo A, Kraeutner S, Tremblay A, Little G, Bardouille T. Laterality of brain activity during motor imagery is modulated by the provision of source level neurofeedback. Neuroimage 2014; 101:159167.

25. Glover S, Dixon P. Context and vision effects on real and imagined actions: Support for the common representation hypothesis of motor imagery. Journal of Experimental Psychology Human Perception \& Performance 2013; 39(5):1352-1364.

26. Lepage J-F, Tremblay S, Dang KN, Champoux F, Théoret $\mathrm{H}$. Action related sounds induce early and late modulations of motor cortex activity. Neuroreport 2010; 21(4):250-253.

27. Rosburg T, Kreitschmann-Andermahr I. The effects of ketamine on the mismatch negativity (MMN) in humans - A meta-analysis. Clinical Neurophysiology Official Journal of the International Federation of Clinical Neurophysiology 2015; 127(2):1387-1394.

28. Näätänen R, Kujala T, Light G. The Mismatch Negativity: A Window to the BrainA Window to the Brain. 2019.

29. Papenberg G, H?Mmerer D, Müller V, Lindenberger U, Li SC. Lower theta inter-trial phase coherence during performance monitoring is related to higher reaction time variability : A lifespan study. Neuroimage 2013; 83(4):912. 
30. Yu L, Wang S, Huang D, Wu X, Zhang Y. Role of inter-trial phase coherence in atypical auditory evoked potentials to speech and nonspeech stimuli in children with autism. Clinical Neurophysiology 2018;

129(7):1374-1382.

\section{Tables}

Table 1. Statistical analysis of EEG power in different brain regions and frequency bands

\begin{tabular}{cccccccccc}
\hline Area & \multicolumn{1}{c}{ Band } \\
\cline { 2 - 11 } & \multicolumn{3}{c}{ alpha } & \multicolumn{4}{c}{ beta } & \multicolumn{3}{c}{ gamma } \\
\cline { 2 - 11 } fp1 & F & df & P-value & F & df & P-value & F & df & P-value \\
cM1 & 0.106 & 1 & 0.464 & 4.561 & 1 & 0.040 & 5.002 & 1 & 0.032 \\
lM1 & 0.722 & 1 & 0.747 & 0.061 & 1 & 0.807 & 1.459 & 1 & 0.235 \\
rM1 & 0.236 & 1 & 0.630 & 4.512 & 1 & 0.041 & 5.508 & 1 & 0.031 \\
lT1 & 0.711 & 1 & 0.405 & 0.060 & 1 & 0.808 & 2.468 & 1 & 0.125 \\
rT1 & 0.154 & 1 & 0.697 & 0.003 & 1 & 0.840 & 1.232 & 1 & 0.275 \\
\hline
\end{tabular}

Statistical analysis in EEG power spectrum obtained by one-way ANOVA. The fpl represent prefrontal lobe, cMl represent parietal motor cortex, lM1 represent left motor parietal lobe, rM1 represent right motor parietal lobe, IT1 represent left auditory temporal lobe and rT1 represent right auditory temporal lobe. Red in the table represents areas with significant differences.

Table 2. The statistical analysis result of MMN.

\begin{tabular}{cccc}
\hline Area & \multicolumn{3}{c}{ MMN } \\
\cline { 2 - 4 } cMl & F & Df & P-value \\
\cline { 2 - 4 } & 6.27 & 1 & 0.017 \\
lM1 & 9.683 & 1 & 0.004 \\
rM1 & 2.057 & 1 & 0.161
\end{tabular}

Statistical analysis in MMN value obtained by one-way ANOVA. The cMl represent parietal motor cortex, lM1 represent left motor parietal lobe, rM1 represent right motor parietal lobe, Red in the table represents areas with significant differences.

Table 3. The statistical analysis result of ITPC.

\begin{tabular}{cccccccccc}
\hline Area & \multicolumn{10}{c}{ Band } \\
\cline { 2 - 11 } & \multicolumn{3}{c}{ alpha } & beta & \multicolumn{3}{c}{ gamma } \\
\cline { 2 - 11 } fp1 & F & df & P-value & F & df & P-value & F & df & -value \\
CM1 & 0.631 & 1 & 0.008 & 2.946 & 1 & 0.095 & 6.238 & 1 & 0.018 \\
lM1 & 11.12 & 1 & 0.433 & 4.330 & 1 & 0.045 & 1.864 & 1 & 0.181 \\
rM1 & 0.139 & 1 & 0.711 & 5.901 & 1 & 0.021 & 8.744 & 1 & 0.006 \\
lT1 & 0.621 & 1 & 0.436 & 1.511 & 1 & 0.227 & 0.095 & 1 & 0.760 \\
rT1 & 0.061 & 1 & 0.807 & 0.000 & 1 & 0.107 & 1.917 & 1 & 0.600 \\
\hline
\end{tabular}


Statistical analysis in ITPC time-frequency superposition obtained by one-way ANOVA. The fpl represent prefrontal lobe, $\mathrm{cMl}$ represent parietal motor cortex, lM1 represent left motor parietal lobe, rM1 represent right motor parietal lobe, IT1 represent left auditory temporal lobe and rT1 represent right auditory temporal lobe. Red in the table represents areas with significant differences.

\section{Figures}

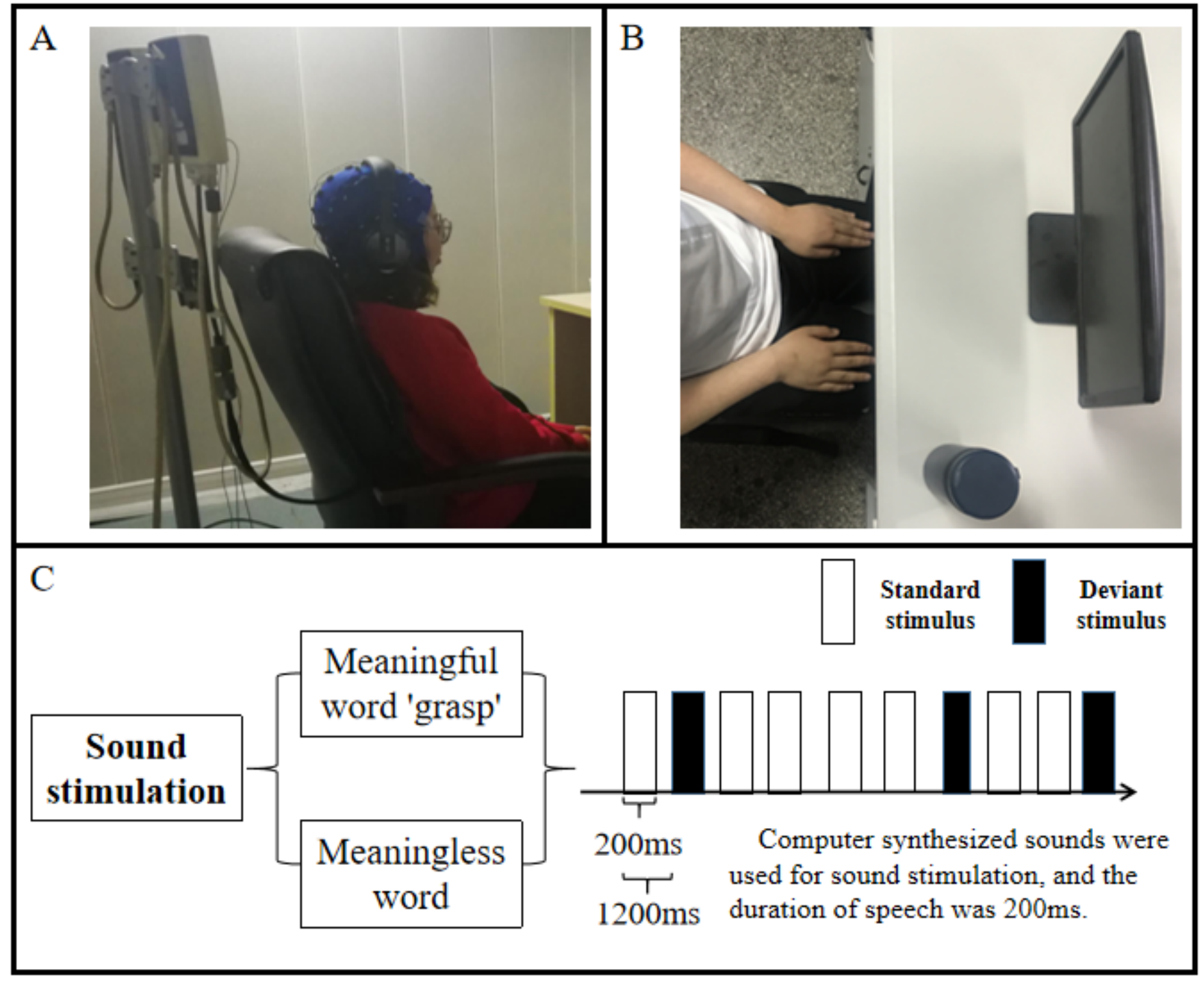

Figure 1

Task design. (A) The picture of the experimental environment, the subjects kept sitting relaxed with their hands flat on their legs. (B) The top view of the experimental environment, the subjects are facing the display device, and the physical cup for right hand movement imagination is placed on the desktop and on the right side of the display. (C) Experimental flow diagram, the experiment was divided into two 
groups. The two groups used the same standard stimulus, and the deviation stimulus used real meaning sound 'grasp' and no real meaning sound respectively. The stimulating sound was composed by computer. The playing time was $200 \mathrm{~ms}$ and the interval was $1200 \mathrm{~ms}$.

\section{$\alpha-b a n d$}

\section{$\beta$-band}

\section{$\gamma$-band}

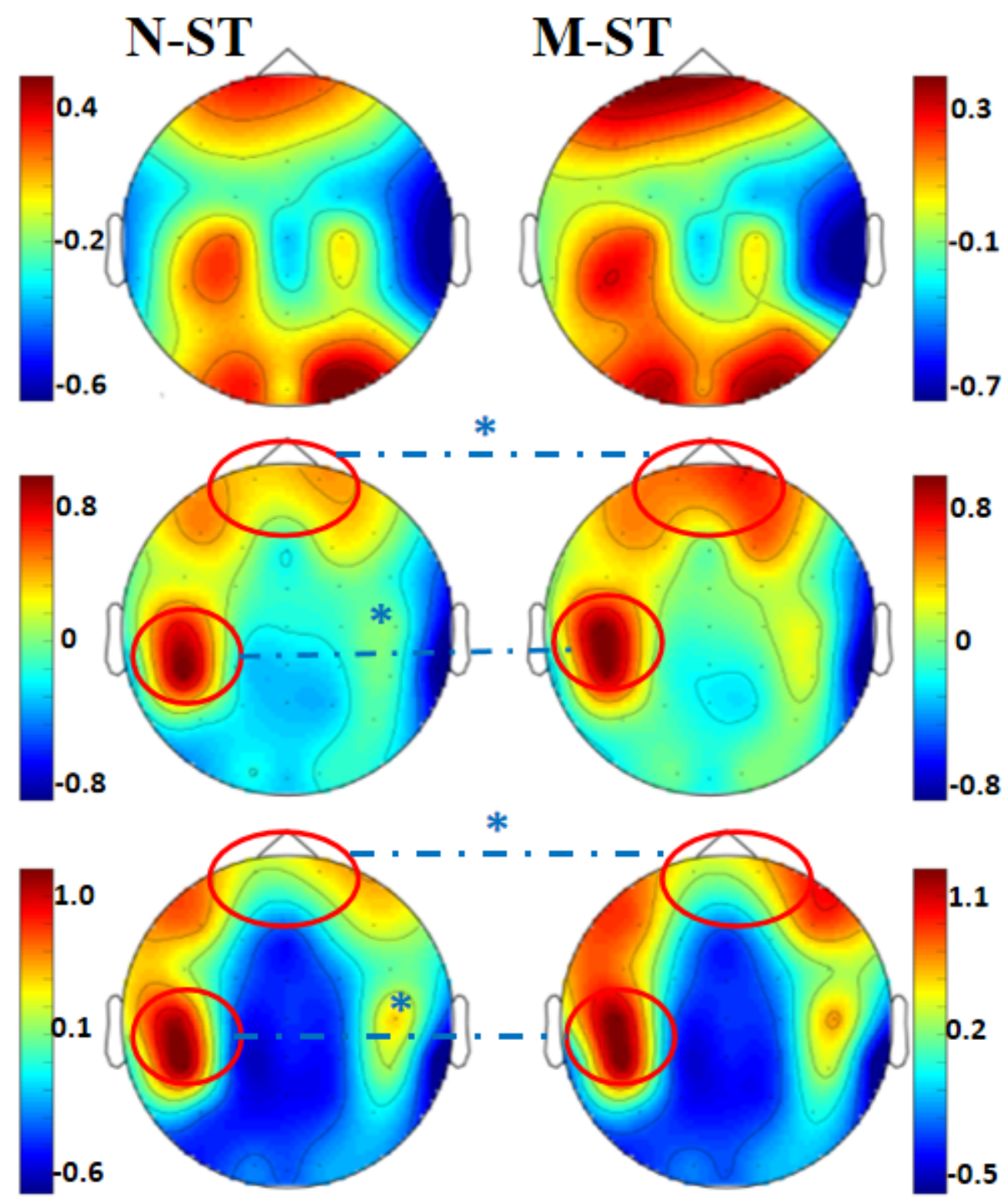

Figure 2

Mean EEG power spectrum of subjects. In the figure, the "N-ST" group is on the left and the "M-ST" group on the right. The EEG power distribution characteristics of $a, \beta$ and $\gamma$ bands are shown respectively. The red circle and " $* "$ indicate the areas with significant differences in power spectrum statistics. It can be seen from the figure that the power produced by the prefrontal cortex and left motor cortex of the "M-ST" group was significantly higher than that of the "N-ST" group. 

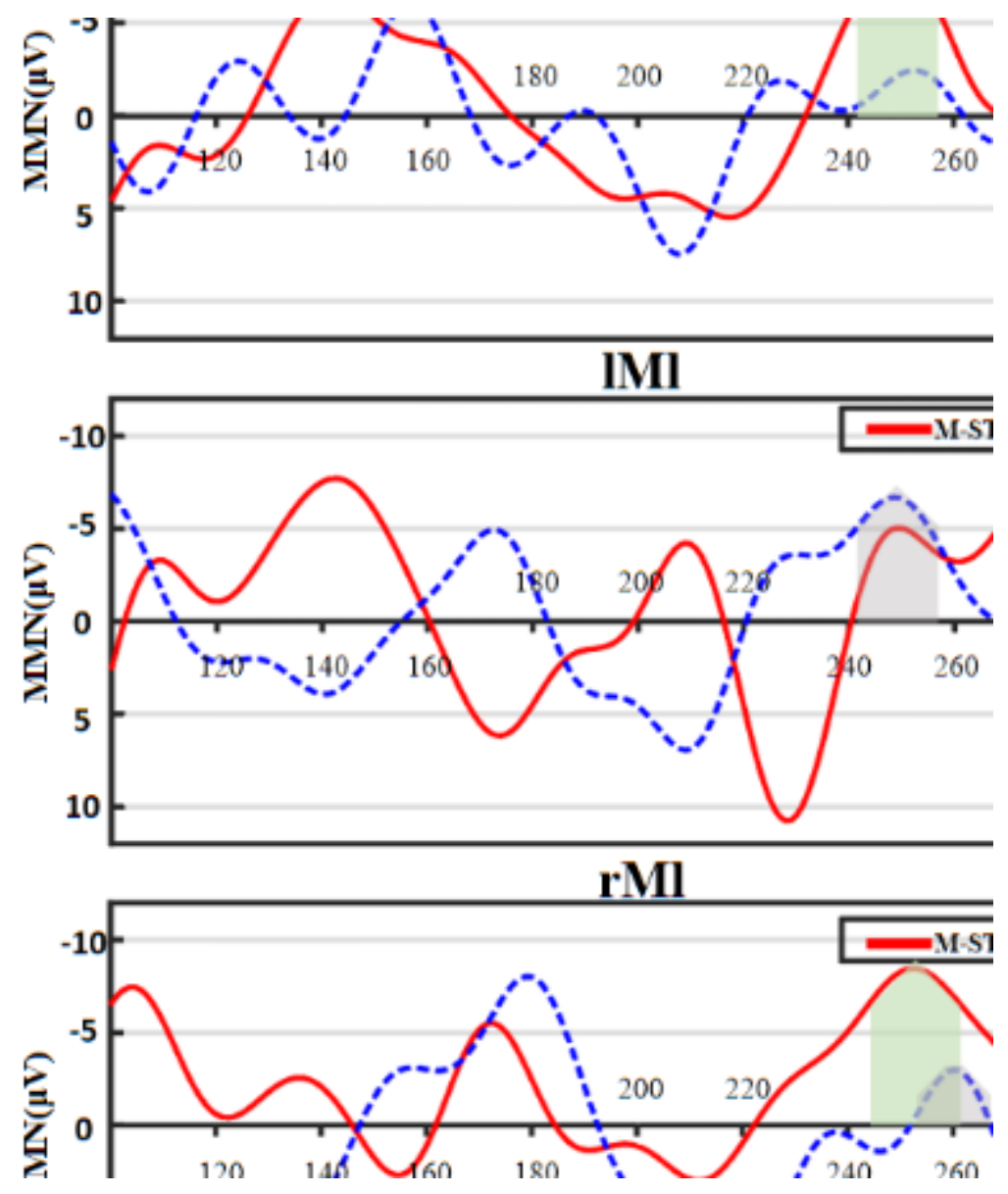

Figure 3

Figure of Mean distribution of MMN. "fPI" represents the prefrontal parietal lobe area, "IMI" represents the left motor cortex, and "rMI" represents the right motor cortex. The red solid line represents the MMN value of group 'M-ST', and the blue dotted line represents the MMN value of group ' $\mathrm{N}-\mathrm{ST}$ '. The light green area and the light gray area represent the superposition range of MMN statistical values of group 'M-ST' and group 'N-ST' respectively. 

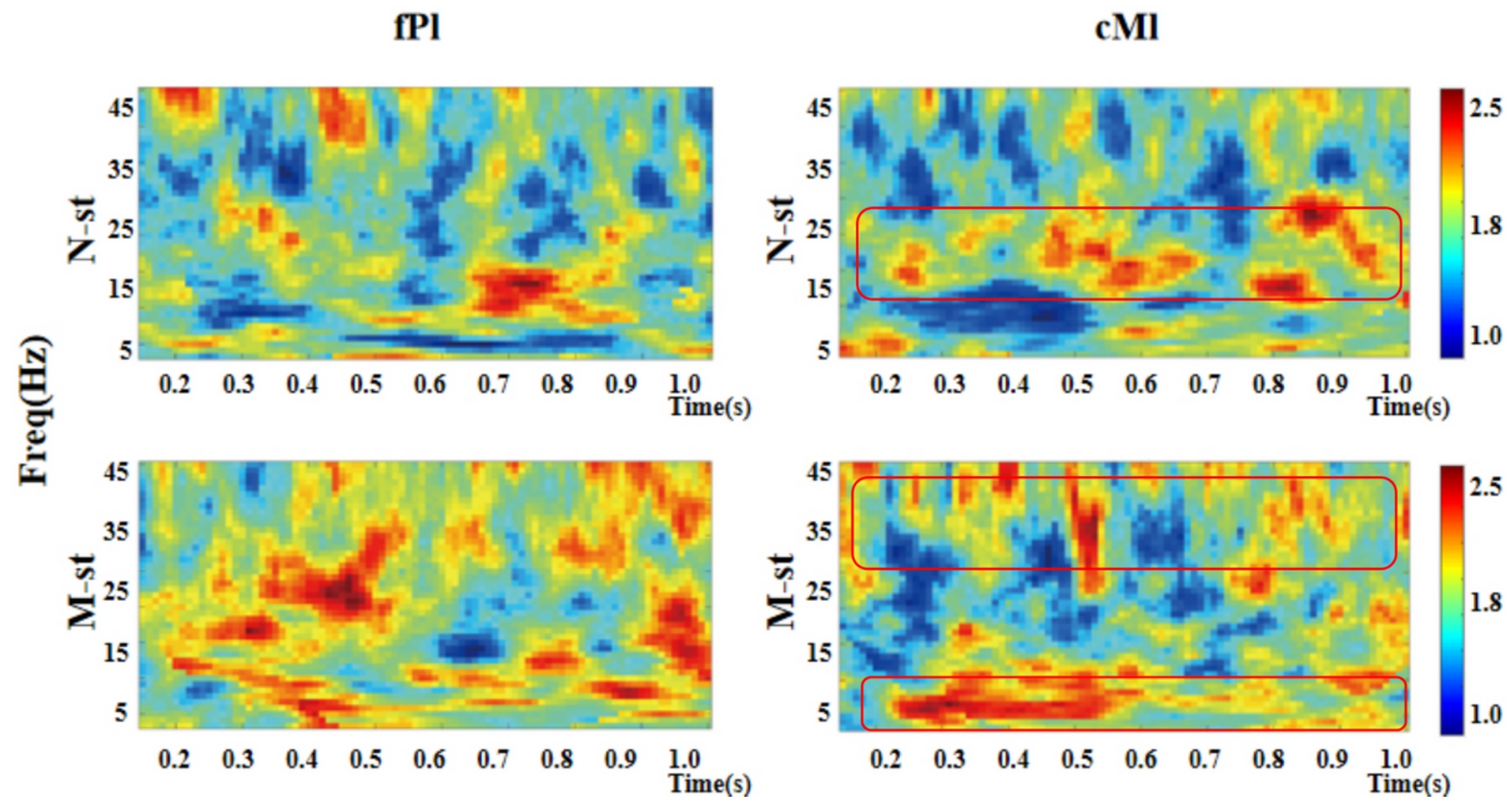

Figure 4

Time-frequency distribution of ITPC in prefrontal and parietal regions. "fPI" represents the prefrontal parietal lobe area, "cMl" represents the parietal motor cortex. "M-st" represents the true meaning sound stimulation group, and the "N-st" band represents the meaningless sound stimulation group. It can be seen from the figure that the ITPC values of the "M-st" group in the frontal parietal lobe region are in a high state in all frequency bands. In the parietal motor cortex, ITPC values of M-st group and n-st group were different. The red boxes represent areas with high ITPC values. 
IMl
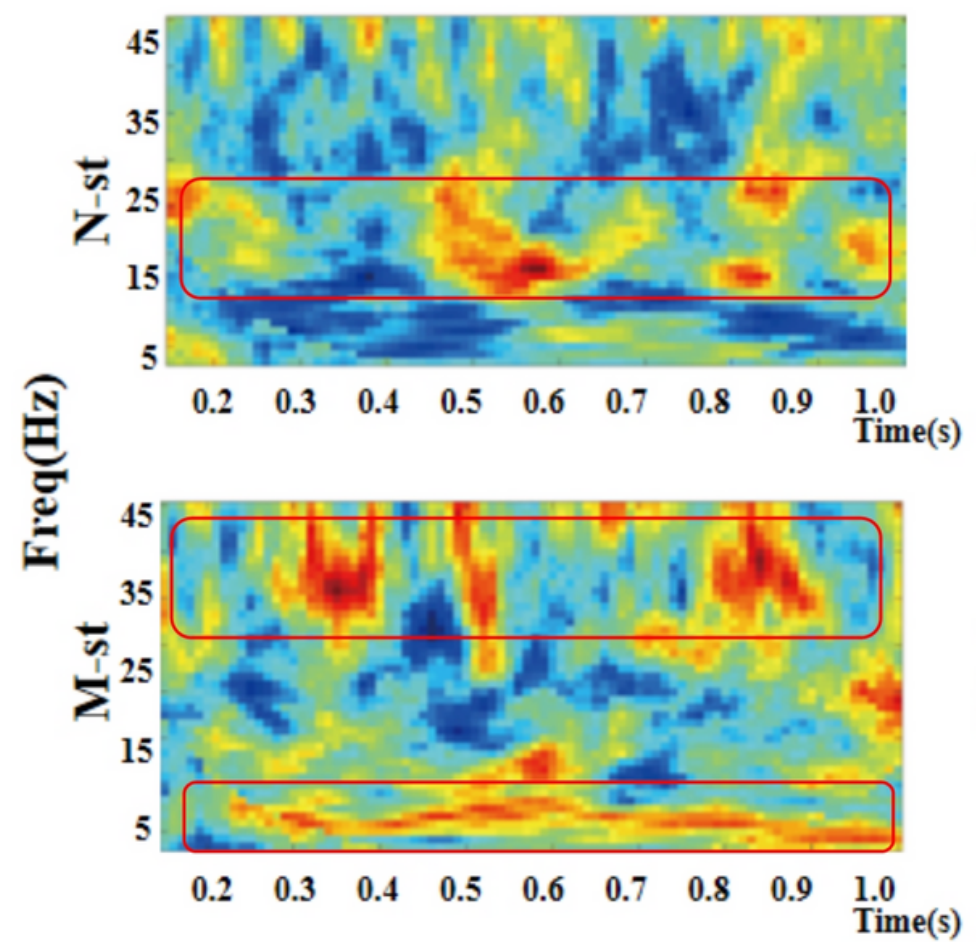

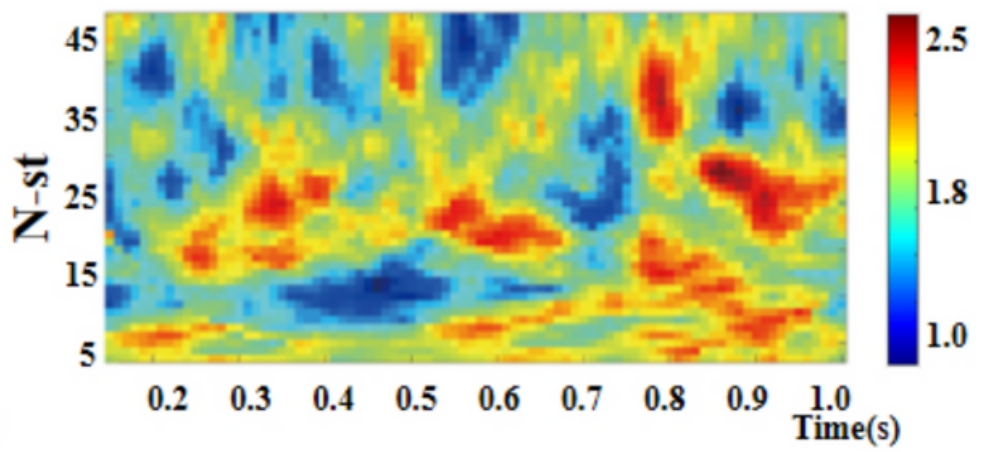

rMl

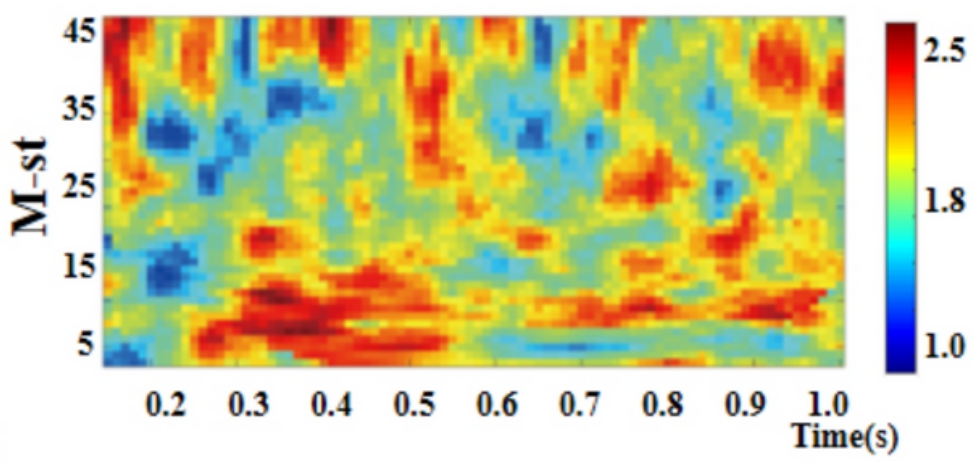

Figure 5

Time-frequency distribution of ITPC in left motor cortex and right motor motor cortex. "IMI" represents the left motor cortex and "rMl" represents the right motor cortex. "M-st" represents the true meaning sound stimulation group, and the " $\mathrm{N}-\mathrm{st}$ " band represents the meaningless sound stimulation group. The distribution of ITPC values in left motor cortex was significantly different. There was no significant difference in the distribution of ITPC in right motor cortex. 
ITI

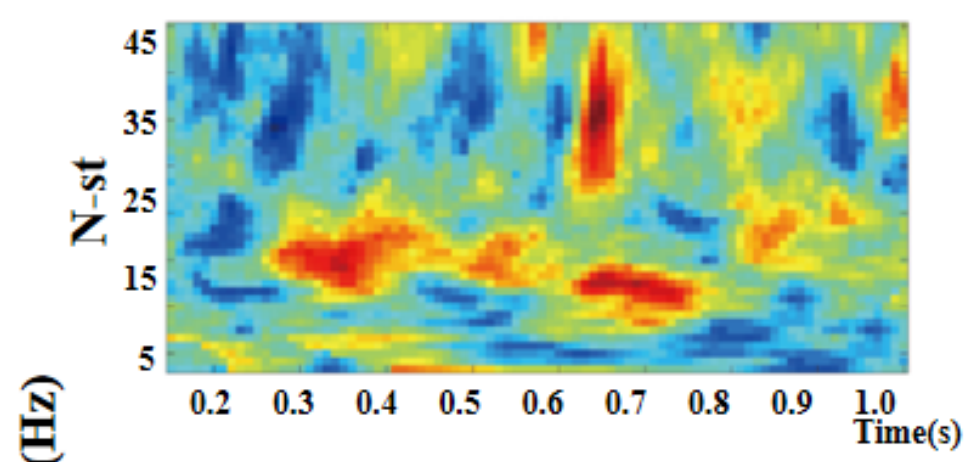

(1)

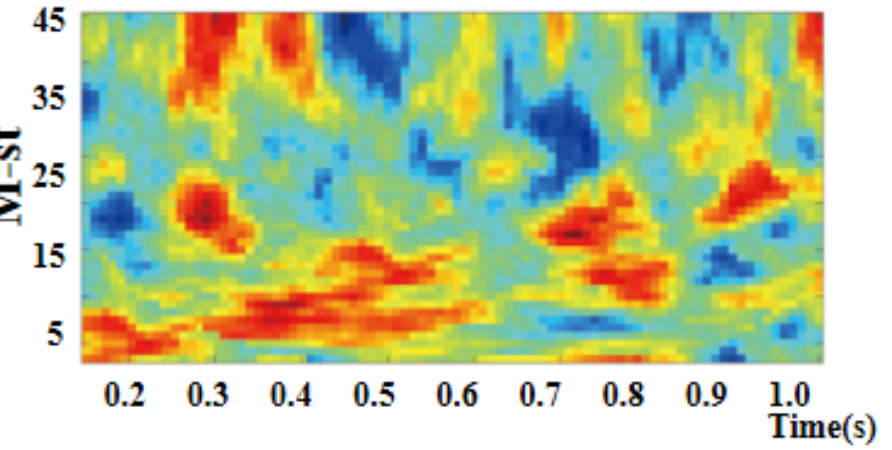

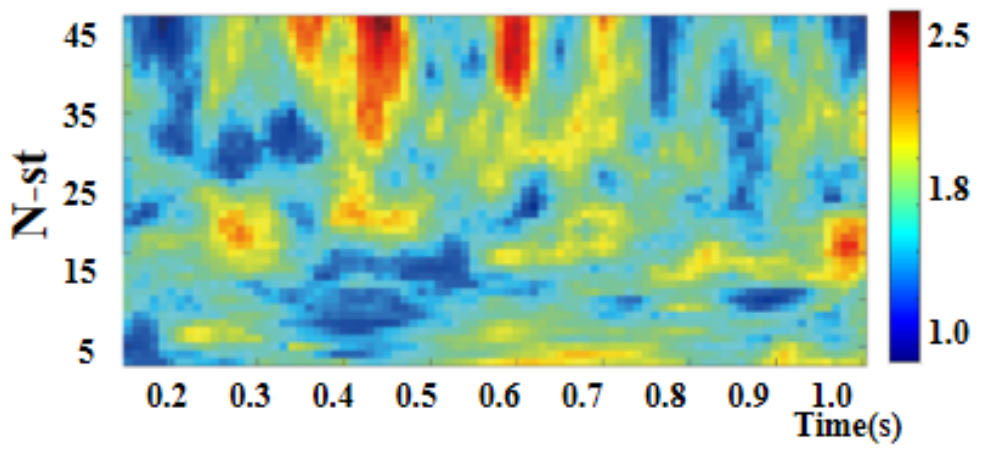

rT1

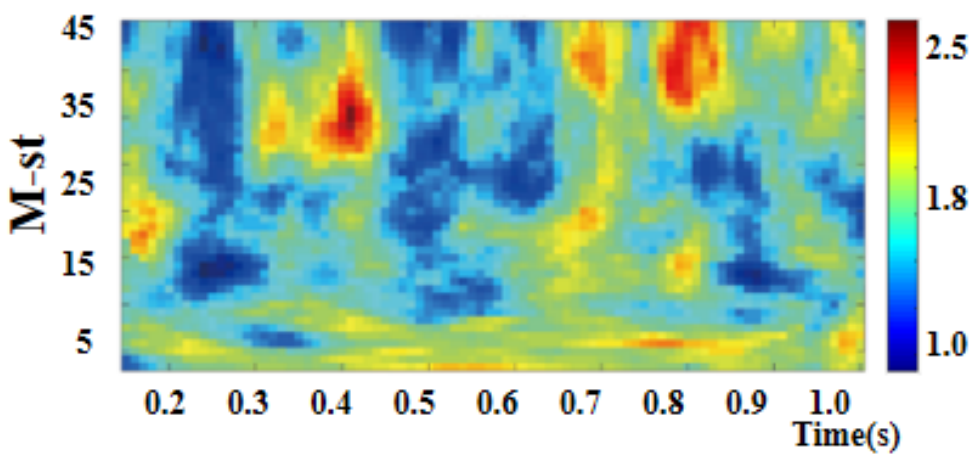

Figure 6

Time-frequency distribution of ITPC in left and right auditory temporal lobes. "ITI" represents the left temporal lobe and "rTI" represents the right temporal lobe. "M-st" represents the true meaning sound stimulation group, and the "N-st" band represents the meaningless sound stimulation group. As can be seen in the figure, the ITPC value of the left temporal lobe is higher than that of the right temporal lobe. 

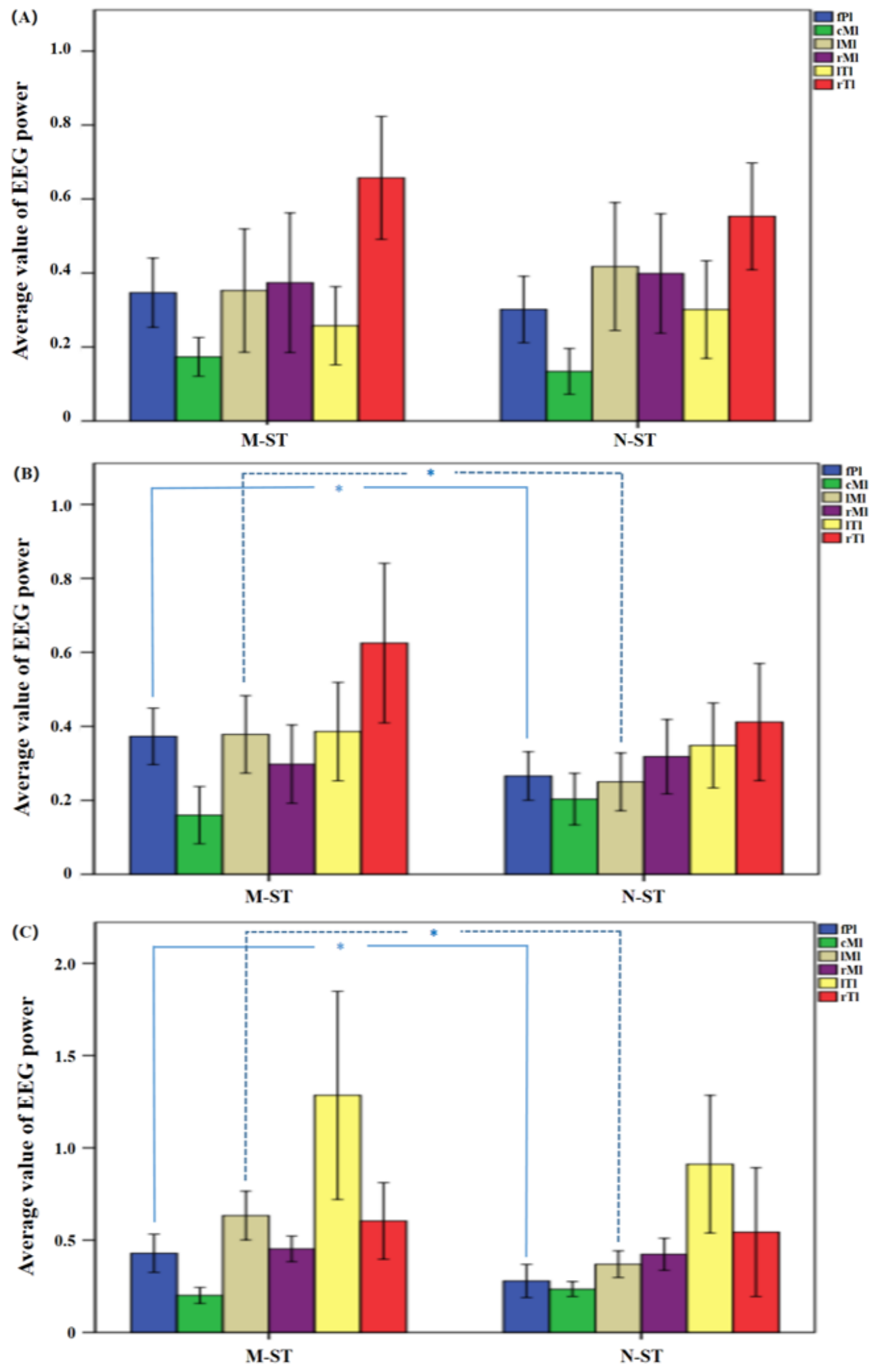

Figure 7

Average normalized EEG power across all the 18 subjects for M-ST task and N-ST task. (A) The mean normalized EEG power within the alpha band. (B) The mean normalized EEG power within the beta band. (C) The mean normalized EEG power within the gamma band. * refers to $p<0.05$ with one-way ANOVA. The statistical results showed that the activity of FPL and LML area was significantly increased in bate band and gamma band. 


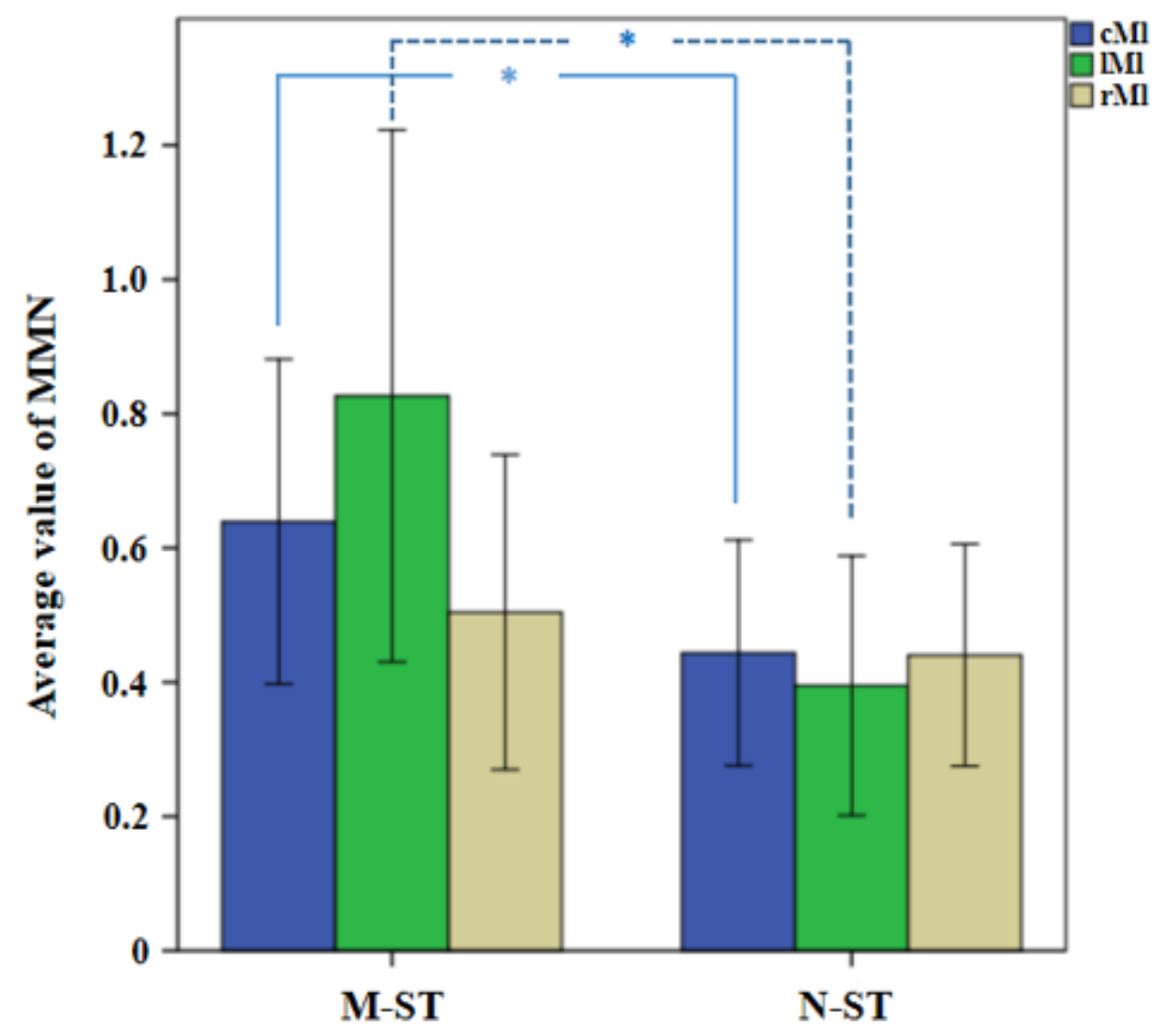

Figure 8

Mean MMN value across all 18 subjects and the results of statistical analysis. * refers to $p<0.05$ with oneway ANOVA. In the M-ST task, the MMN from the frontal parietal motor sensory cortex and the left motor cortex was significantly enhanced. 

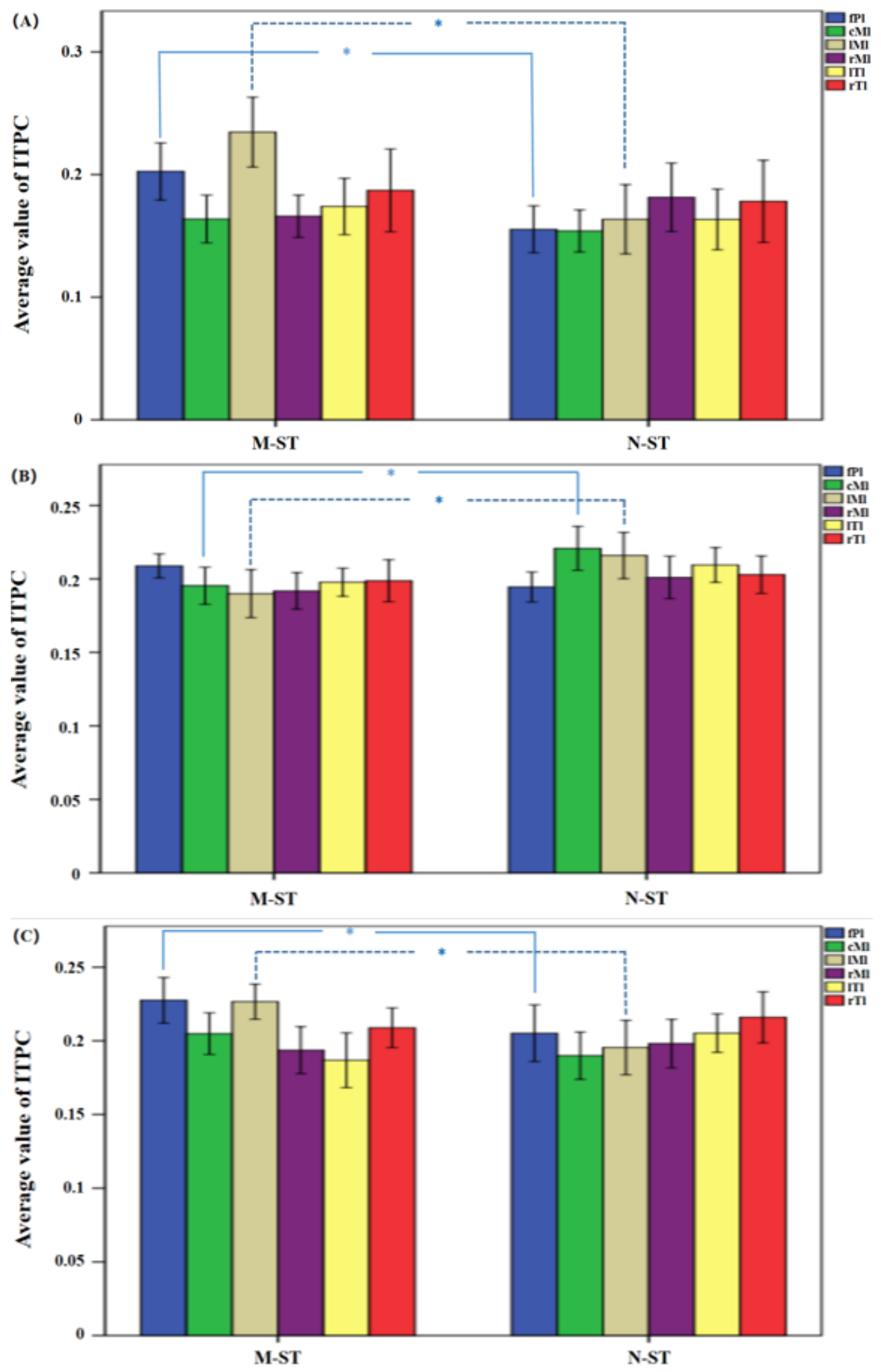

Figure 9

The mean ITPC values of 18 subjects in different brain regions and different frequency bands. (A) The mean value of ITPC within the alpha band. (B) The mean value of ITPC within the beta band. (C) The mean value of ITPC within the gamma band. * refers to $p<0.05$ with one-way ANOVA. The results of statistical analysis showed that the ITPC values of prefrontal cognitive cortex and left parietal motor cortex in M-ST group were significantly higher than those in N-ST group in alpha frequency band, the 
ITPC values of parietal motor cortex and left parietal motor cortex of N-ST group were significantly higher than those of M-ST group under bate frequency band, and ITPC values of prefrontal cognitive cortex and left parietal motor cortex of M-ST group under gamma band were significantly higher than those of N-ST group. 